\title{
Error analysis of contactless optical temperature probing methods for cryogenic Yb:YAG
}

\author{
Umit Demirbas $^{1,2}$ (1) $\cdot$ Jelto Thesinga ${ }^{1} \cdot$ Martin Kellert $^{1} \cdot$ Simon Reuter $^{1} \cdot$ Franz X. Kärtner $^{1,3,4} \cdot$ Mikhail Pergament $^{1}$
}

Received: 14 May 2021 / Accepted: 5 July 2021 / Published online: 13 July 2021

(c) The Author(s) 2021

\begin{abstract}
In this work, we have investigated six different in situ optical contactless temperature probing methods for cryogenic Yb:YAG systems. All the methods are based on variation of fluorescence spectra with temperature, and they either look at the width of the emission line, the ratio of the emission intensity at different wavelengths and to the overall spectral change at selected wavelength intervals. We have shown that, for Yb:YAG crystal with homogeneous temperature distribution, one can perform real-time contactless optical temperature measurements with $a \pm 1 \mathrm{~K}$ accuracy in the $78-300 \mathrm{~K}$ range. We have further tested the methods in measuring the average temperature of Yb:YAG rods at up to $500 \mathrm{~W}$ absorbed pump power level. We have seen that, a real-time temperature measurement accuracy of $\pm 5 \mathrm{~K}$ is feasible in both lasing and non-lasing situations for estimating the average temperature of crystals under nonhomogeneous thermal load. The techniques are quite valuable in evaluating the bonding quality of Yb:YAG crystals in cryogenic systems. Moreover, the real-time temperature information provides feedback on parameters like cavity alignment status and extraction efficiency to the laser engineers while optimizing the system.
\end{abstract}

\section{Introduction}

Yb-doped cryogenic solid-state lasers and amplifiers such as $\mathrm{Yb}$ :YAG and $\mathrm{Yb}$ :YLF have reached multi-100 $\mathrm{W}$ to $\mathrm{kW}$ average power levels over the last decades [1-9]. Further power scaling of these systems require detailed understanding of thermal effects and measurement/estimation of internal crystal temperatures during laser/amplifier operation [10-14]. Infrared thermal cameras that could effectively provide temperature information for room-temperature systems [15] are not effective at cryogenic temperatures. Several different methods that are mostly based on measurement

Umit Demirbas

uemit.demirbas@cfel.de

1 Center for Free-Electron Laser Science, Deutsches Elektronen-Synchrotron DESY, Notkestraße 85, 22607 Hamburg, Germany

2 Laser Technology Laboratory, Department of Electrical and Electronics Engineering, Antalya Bilim University, Antalya, Turkey

3 Physics Department, University of Hamburg, Luruper Chaussee 149, 22761 Hamburg, Germany

4 The Hamburg Centre for Ultrafast Imaging, Luruper Chaussee 149, 22761 Hamburg, Germany of emission spectrum had already been proposed for estimating laser crystal temperature. Petit et al. [16] used the temperature variation of upconversion spectra from the $\mathrm{Er}$ impurities in the 460-580 $\mathrm{nm}$ region in Yb:CALGO samples in estimating temperatures in the $50-120^{\circ} \mathrm{C}$ range. A similar method is used in estimating temperatures of a $\mathrm{Er}: \mathrm{Yb}: \mathrm{LuAB}$ microchip laser in the 300-550 K interval [17]. The slight shift of laser wavelength was used for temperature estimation in the $20-50{ }^{\circ} \mathrm{C}$ range in a diode-pumped $\mathrm{Yb}$ :YAG laser system [18]. Fruse et al. [19, 20] demonstrated that the fluorescence intensity ratio of $\mathrm{Yb}: \mathrm{YAG}$ at $1022 \mathrm{~nm}$ and $1027 \mathrm{~nm}$ could be used for estimation of temperature in the 77-200 K area. Chi et al. $[10,21]$ used the fluorescence spectra of $\mathrm{Yb}$ :YAG in the 1015-1029 nm interval for estimating temperatures in the 50-140 K range using neural network functions. The variation of pump absorption at $1030 \mathrm{~nm}$ with temperature was employed in estimating Yb:YAG thin-disk temperatures in the 40-300 K range by Petrov et al. [22]. We have recently investigated different spectral methods for temperature estimation of cryogenic Yb:YLF samples and demonstrated a temperature estimation accuracy below $\pm 1 \mathrm{~K}$ in the 78-300 $\mathrm{K}$ region [23], and used this for analyzing laser performance $[24,25]$. Similar methods had already been in use for temperature estimation of $\mathrm{Yb}$ :YLF and $\mathrm{Yb}$ :YAG by the cryogenic optical refrigeration community [26-30]. 
In our work, while developing high-power cryogenic Yb:YAG systems that are based on rods and thin disks, a contactless method to probe the bonding quality of the gain elements to the heat sink was required as this is very critical for laser/amplifier performance [31-33]. Furthermore, while optimizing the laser/amplifier, a real-time temperature information is quite valuable for the laser engineer/scientist to pinpoint the issues in the system. For both cases, a temperature estimation accuracy of at least $\pm 5 \mathrm{~K}$ is necessary.

Motivated by that, in this work, we have performed a set of detailed experiments, where we have comparatively investigated pros and cons of different temperature estimation methods for $\mathrm{Yb}$ :YAG systems operating near cryogenic temperatures. In the analysis, we have used six different methods for temperature estimation that are based on investigation of: (1) the FWHM of the main $\sim 1030 \mathrm{~nm}$ line, (2) the ratio of the main $\sim 1030 \mathrm{~nm}$ peak intensity to the $\sim$ $1027 \mathrm{~nm}$ valley intensity, (3) the ratio of the $\sim 1024.5 \mathrm{~nm}$ side peak to the $\sim 1027 \mathrm{~nm}$ valley, and (4) the ratio of the $1022 \mathrm{~nm}$ side peak to the $\sim 1027 \mathrm{~nm}$ valley (as also used in $[19,20])$. We have also considered the differential luminescence thermometry (DLT) method, where we looked at the spectral changes in wider spectral intervals, focusing on the (5) 950-1060 nm and (6) 950-1025 nm regions. We have shown that, with all the methods, a temperature estimation accuracy below $\pm 4 \mathrm{~K}$ is feasible. The DLT method is more demanding in terms of time and effort, since it requires a more careful calibration process compared to other methods. On the other hand, it provides better temperature estimation accuracy (below $\pm 1 \mathrm{~K}$ ) compared to the other simpler methods. Among the fast methods, we have seen that $1024.5 / 1027 \mathrm{~nm}$ and $1022 / 1027 \mathrm{~nm}$ intensity ratio provides a performance close to DLT (below $\pm 1.2 \mathrm{~K}$ ).

The paper is organized as follows: Sect. 2 presents the experimental setups. In Sect. 3, we provide details of the different methods used in temperature estimation, and in Sect. 4, we present sample reference fluorescence data and the calibration curves. Then in Sect. 5, we analyze the

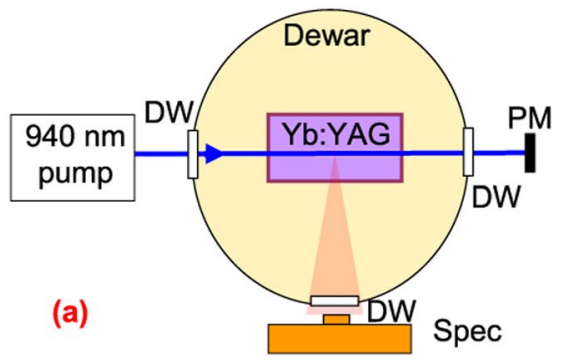

Fig. 1 a Schematic of the contactless crystal temperature measurement setup used for Yb:YAG. The crystal is indium soldered to a pyramidal copper heat sink element, which is in direct contact with boiling liquid nitrogen. The fluorescence signal is collected via a dewar window placed 90 degrees to pump beam direction. Dimen- temperature estimation accuracy of each method. Sample temperature measurements for lasing and non-lasing Yb:YAG laser rod systems are given in Sect. 6. Finally, in Sect. 7, we summarize with a brief discussion.

\section{Experimental setup}

We have tested several methods for temperature estimation of $\mathrm{Yb}: \mathrm{YAG}$, and all methods were based on the measured variation of emission spectra of Yb:YAG with temperature. Figure 1a shows a simple schematic of the emission measurement setup. A fiber-coupled, $940 \mathrm{~nm}$ laser diode with an $M^{2}$ of around 220 was used as the excitation source. The diode output is collimated with a $72 \mathrm{~mm}$ focal length lens, and focused to a pump diameter of $2.08 \mathrm{~mm}$ using a $250 \mathrm{~mm}$ lens. The pump had a flattop beam profile and was imaged to the center of the crystal. A $1 \%$ Yb-doped YAG crystal with a $24 \mathrm{~mm}$ length and $5 \times 15 \mathrm{~mm}^{2}$ aperture was used in the experiments. The Yb:YAG crystal is indium soldered from the top side to a multi-stage pyramidal cold head (Fig. 1b). Real-time measurement of cold head temperature with $\pm 0.1 \mathrm{~K}$ accuracy was achieved via thermal sensors connected to the cold head.

For the temperature-dependent emission measurements, to collect the reference data, the dewar containing the Yb:YAG crystal is filled with liquid nitrogen. Later, once the nitrogen in the dewar tank is evaporated, the bulky dewar interior mass including the cold finger and the $\mathrm{Yb}$ :YAG crystal head heats up very slowly in around $10 \mathrm{~h}$. In this heating-up cycle, the Yb:YAG crystal and the cold head are in thermal equilibrium, and the crystal temperature could be accurately measured using the thermal sensors. Emission measurements were made with little average power load on the crystal to preserve the thermal equilibrium between the crystal and the cold head (1 ms long pump pulses with $1 \mathrm{~kW}$ of peak power at $0.2 \mathrm{~Hz}: \sim 200 \mathrm{~mW}$ average absorbed power, around $20 \mathrm{~mW}$ thermal load).

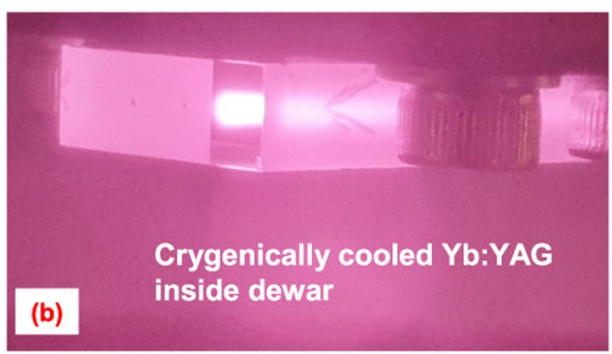

sions are not to scale. $D W$ Dewar window, Spec spectrometer, $P M$ power meter. b A photo of the cryogenically cooled Yb:YAG crystal inside the dewar while being pumped with hundreds of Watts of diode power at $940 \mathrm{~nm}$ 
The reference emission spectra were measured at a $90^{\circ}$ angle to the pump propagation direction via a window at the side of the dewar (Fig. 1a). An Ocean Optics HR4000 spectrometer with a spectral resolution of $0.1 \mathrm{~nm}$, and a spectral coverage from 900 to $1060 \mathrm{~nm}$ was used in the measurements. The spectrometer contained a 3648 pixel CCD array, and spectral data are taken with $\sim 0.045 \mathrm{~nm}$ steps ((1060-900)/3648). The spectrometer had a very narrow entrance slit with a size of around $5 \mu \mathrm{m}$ by $2 \mathrm{~mm}$. The entrance slit was hidden $10 \mathrm{~mm}$ deep inside a cylindrical metallic entrance with a dimeter of $3 \mathrm{~mm}$. This narrow entrance blocked stray light and provided directionality. In the experiments, the spectrometer entrance was aligned to look at the central portion of the crystal. Due to the large dewar mechanics, the spectrometer was around $15 \mathrm{~cm}$ away from the crystal, but a good signal-to-noise ratio could still be achieved without any additional imaging element. To improve our error analysis accuracy, a total of 630 emission measurements were made in the temperature range of 78-300 K: roughly one measurement at every $0.35 \mathrm{~K}$. We estimate a maximum temperature measurement error of $\pm 0.25 \mathrm{~K}$ for these data.

Note that the reference data should be taken with great care to minimize temperature estimation errors. In general, the reference spectrum should be collected very carefully in a geometry similar to the intended use of the Yb:YAG sample in the laser/amplifier experiments. This is due to various effects such as self-absorption and amplified spontaneous emission, the reference data might vary easily with: (1) the doping of the crystal used [23], (2) the position of the excitation beam with respect to crystal, (3) the intensity of the excitation beam, (4) the size of the probe beam used, (5) the reference data signal collection geometry (system etendue), and also (6) the spectrometer instrument settings and specifications. For this work, where the specific aim is to accurately estimate the temperature of rod-type laser crystals under thermal load, we have collected the reference data using Yb:YAG crystals with doping adequate for lasing in rod geometry and the emission collection geometry is chosen to be exactly the same as the one that is used in laser experiments. We have further used the same pump beam size and pump beam position as in laser experiments (as seen in Fig. 1b) to minimize temperature estimation errors.

Once we have collected temperature-dependent reference emission data and prepared the calibration curves, the system was cooled down again by refilling liquid nitrogen, and the crystal was pumped with the pump module operating in continuous-wave $(\mathrm{cw})$ regime, where incident cw pump powers up to $500 \mathrm{~W}$ were applied to the $\mathrm{Yb}$ :YAG crystal. The untouched emission setup was used to measure the emission at different pump power levels, and the collected data are used to predict the temperature of the Yb:YAG crystals under thermal load. At these power levels, due to pump saturation, the pump light could penetrate deeper inside the gain element, and this provides a relatively homogenous heating. As mentioned, the spectrometer input was directed toward the center of the crystal, and hence, the spectral data collected provide a rough measurement of average temperature of the Yb:YAG sample. In this method, it is also possible to adjust the spectrometer slit entrance direction to the front and back parts of the crystal to obtain a rough 1-D scan of temperature profile. We should note here that, due to the relatively large dewar that was used in our experiment, the spectrometer entrance was around $15 \mathrm{~cm}$ away from the laser crystal. As a result, besides the directly emitted light from the excited volume of interest, scattered light from other regions could still reach the spectrometer and this reduces the spatial resolution of temperature measurement. More advanced techniques such as confocal laser scanning microscopy geometry could ideally be adopted for 3-D temperature mapping of the crystal with high spatial resolution $(\sim 100-200 \mu \mathrm{m})$ at the expense of increased complexity [10]. In this work, we focused our attention on analyzing the accuracy of different temperature estimation methods, and knowledge of crystal average temperature is sufficient for determining crystal bonding quality or for analyzing laser/amplifier performance for our case.

We have also investigated the usability of the developed temperature estimation methods during lasing experiments. For that, a simple continuous-wave lasing cavity is configured (Fig. 2). A compact flat-flat cavity was employed which consisted of a flat dichroic mirror (DM) and a flat output coupler with a separation of around $30 \mathrm{~cm}$. The DM had a reflectivity higher than $99.9 \%$ in the 990-1040 nm range, and a transmission $>95 \%$ for the pump wavelength. The temperature estimation data are taken while monitoring the Yb:YAG fluorescence spectra via the side window, as it is described earlier.

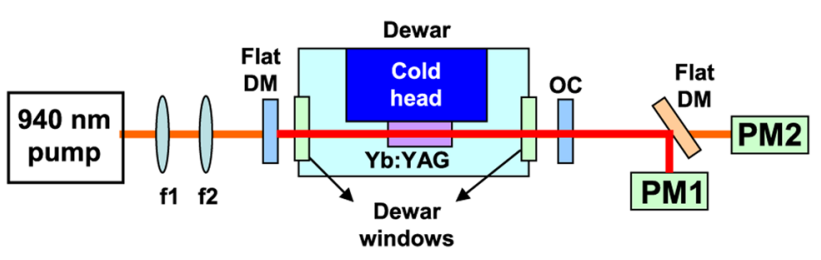

Fig. 2 Schematic of the diode-pumped cryogenic Yb:YAG laser system that is used in continuous-wave lasing experiments. The $30 \mathrm{~cm}$ long simple cavity consists of one-flat dichroic mirror (DM) and a flat $25 \%$ transmitting output coupler (OC). $f 1-f 2$ Lenses for pump coupling, $P M 1-2$ power-meters 


\section{Methods used for temperature probing}

Figure $3 \mathrm{a}$ shows the measured emission spectra of $\mathrm{Yb}$ :YAG at selected temperatures for temperatures ranging from 78 and $300 \mathrm{~K}$ in normalized (arbitrary) units. Figure $3 \mathrm{~b}$ shows the emission spectra near the $\sim 1030 \mathrm{~nm}$ region where the measured variation with temperature is largest. Clearly, the measured spectral shape is a strong function of temperature and could be used for temperature estimation.

To formally attack the problem, one can directly calculate the spectral intensity change at different wavelengths $\left(\Delta S\left(\lambda, T, T_{0}\right)\right)$ with respect to a reference spectrum at a chosen temperature using [27]
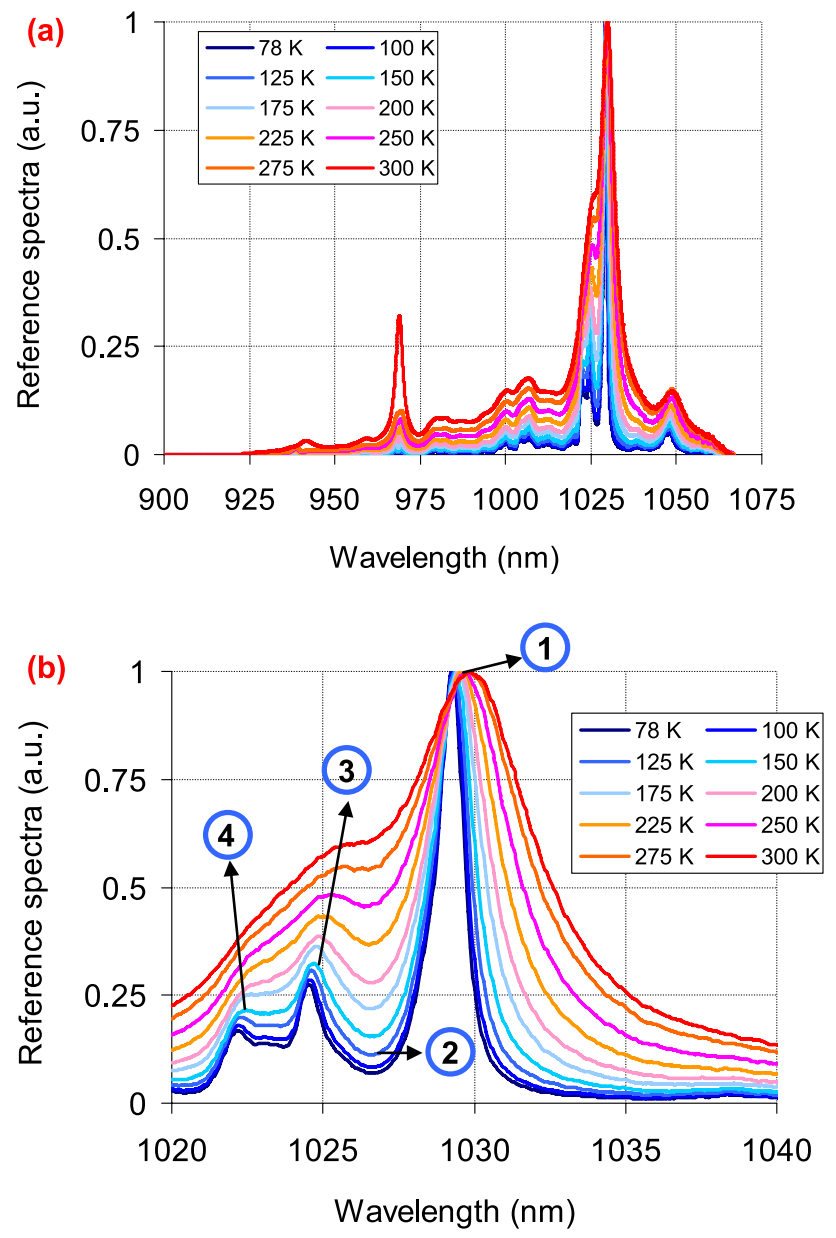

Fig. 3 a Measured normalized reference emission spectra of $1 \%$-doped Yb:YAG as a function of temperature between 78 and $300 \mathrm{~K}$. b A closer look at temperature induced changes in emission spectra in the 1020-1040 $\mathrm{nm}$ wavelength range. In the temperature estimation analysis, ratios of fluorescence intensities at points $1(\sim$ $1030 \mathrm{~nm}), 2(\sim 1027 \mathrm{~nm}), 3(\sim 1024.5 \mathrm{~nm})$, and $4(\sim 1022 \mathrm{~nm})$ are used
$\Delta S\left(\lambda, T, T_{0}\right)=\frac{S(\lambda, T)}{\int S(\lambda, T) d \lambda}-\frac{S\left(\lambda, T_{0}\right)}{\int S\left(\lambda, T_{0}\right) d \lambda}$.

Here $T$ is the temperature, $T_{0}$ is the reference spectra temperature, $\lambda$ is the wavelength, $S(\lambda, T)$ is the measured emission spectra at different temperatures, and the integration limit covers the spectral range of interest. We take the reference temperature as $78 \mathrm{~K}$, since we are more interested in estimating temperatures at cryogenic range. Figure 4 then shows the calculated spectral change $\left(\Delta S\left(\lambda, T, T_{0}\right)\right)$ with respect to the measured $78 \mathrm{~K}$ spectrum $\left(T_{0}=78 \mathrm{~K}\right)$, which confirms that most of the spectral change is occurring near the $1030 \mathrm{~nm}$ transition.

As expected, at elevated temperatures with increasing phonon energy, the emission lines get broader, and the emission spectra become smoother. As one can see (from
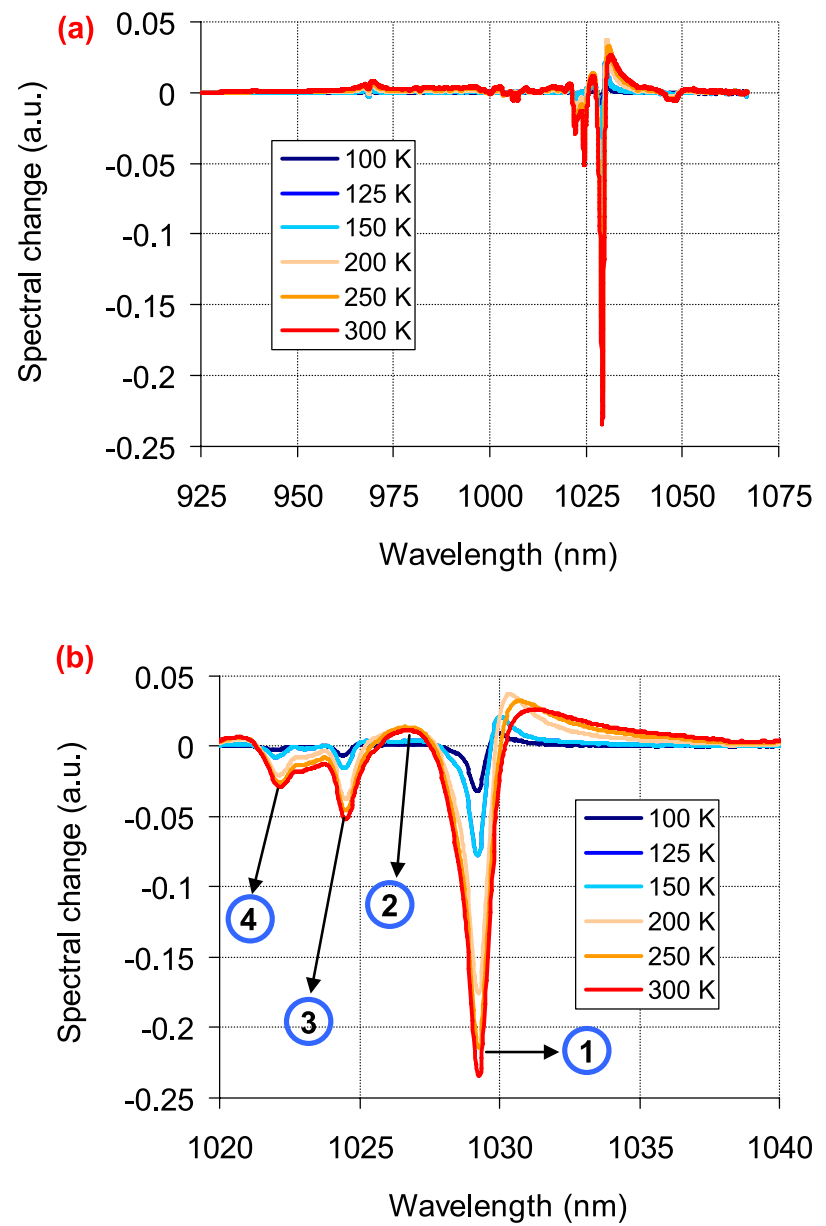

Fig. 4 a Calculated spectral intensity change $\left(\Delta S\left(\lambda, T, T_{0}\right)\right)$ of Yb:YAG crystal compared to a reference spectrum taken at a temperature of $78 \mathrm{~K}$ (normalized for the $935-1067 \mathrm{~nm}$ region); b a closer look at temperature induced spectral change around the main emission peak at $1030 \mathrm{~nm}$ 
Figs. $3 b$ and $4 b$ ), with increasing temperature, the sharp emission peak around $\sim 1030 \mathrm{~nm}$ widens out with heating (point 1 in the figure). Moreover, the spectral dip (around $1027 \mathrm{~nm}$, point 2 in the figure) between these peaks fills in slowly. There are two side peaks located around $\sim 1024.5 \mathrm{~nm}$ and $\sim 1022 \mathrm{~nm}$ (points 3 and 4), that also widens out and smoothens with heating.

It is clear that the measured spectral change in emission could be used for estimation of the temperature, and it is interesting to find out pros and cons of different methods and their error margins. Ideally, for the most accurate temperature estimation, one should look at the temperature induced change in the whole emission spectra, and as mentioned earlier this method is known as DLT (Differential Luminescence Thermometry). On the other hand, this method could not be used in case of lasing or strong amplified spontaneous emission, and requires careful reference data acquisition, and hence, it is time and effort costly. Hence, we have also tried and investigated temperature prediction accuracy of other methods in this work.

The following six methods are investigated/compared for temperature estimation:

1) $1030 \mathrm{~nm}$ peak method: we look at the ratio of the $\sim 1030 \mathrm{~nm}$ emission peak to the $\sim 1027 \mathrm{~nm}$ emission valley (1030/1027 $\mathrm{nm}$ ratio: ratio of intensity around point 1 to point 2, in Fig. 3).

2) $1024.5 \mathrm{~nm}$ peak method: we utilize the ratio of the $\sim 1024.5 \mathrm{~nm}$ emission peak to the $\sim 1027 \mathrm{~nm}$ emission valley $(1024.5 / 1027 \mathrm{~nm}$ ratio: ratio of intensity around point 3 to point 2, in Fig. 3).

3) $1022 \mathrm{~nm}$ peak method: we use the ratio of the $\sim 1022 \mathrm{~nm}$ emission peak to the $\sim 1027 \mathrm{~nm}$ emission valley $(1022 / 1027 \mathrm{~nm}$ ratio: ratio of intensity around point 4 to point 2, in Fig. 3).

4) FWHM method: we have considered the temperature dependence of the full-width half-maximum of the main $\sim 1030 \mathrm{~nm}$ transition.

5) DLT method: we look at the integrated absolute change in the overall spectral shape for temperature estimation. In terms of spectral intervals, we have chosen 950-1060 $\mathrm{nm}$ and 950-1025 nm intervals. The 950$1060 \mathrm{~nm}$ range (DLT-1) covers almost all the emission spectra except the lower wavelength range, which is not included to prevent noise due to the scattered pump signal. In the second interval (DLT-2: 950-1025 nm), we have excluded the main $1030 \mathrm{~nm}$ emission to investigate temperature estimation problems that might be induced by amplified spontaneous emission process.

As a final note, the peak and dip positions mentioned above are also temperature-dependent: as an example, the $\sim 1030 \mathrm{~nm}$ line is located around $1029.25 \mathrm{~nm}$ at $78 \mathrm{~K}$ and moves to around $1030.05 \mathrm{~nm}$ at room temperature as it is clearly visible from Fig. 3b. In our work, as an example, while determining the1030/1027 $\mathrm{nm}$ ratio, we have searched for the exact peak and dip positions around $1030 \mathrm{~nm}$ and $1027 \mathrm{~nm}$, and used intensities at these peak/dip wavelengths (rather than using intensity ratios at fixed wavelength positions).

\section{Reference data for different temperature estimation methods}

In the earlier section, we have introduced the different temperature estimation methods we wanted to investigate. In this section, we will present reference data or calibration curves we have taken for the temperature estimation. As outlined in the experimental section earlier, the reference spectrum should be collected very carefully in a geometry similar to the intended use of the Yb:YAG sample to minimize temperature estimation errors. Hence, the reference data provided here are specific to our geometry and could be slightly different for other configurations.

To start with, Fig. 5a shows the measured variation of the ratio of fluorescence intensity at the emission peaks of $1022 \mathrm{~nm}, 1024.5 \mathrm{~nm}$, and $1030 \mathrm{~nm}$ to the emission dip/ valley at $1027 \mathrm{~nm}$. As we saw in Figs. 3b and 4b as well, with increasing temperature, the emission strength in these peaks diminish as the transitions gets wider with increasing temperature and phonon energy. Hence, all the peaks lose strength (the ratios decrease) with increasing temperature. The change of intensity with temperature is larger for the main $1030 \mathrm{~nm}$ peak. One might think this then could provide the minimal temperature estimation error (due to larger slope with temperature), but this was not the case as it will be shown in the next section. Note also that, above $200-250 \mathrm{~K}$, the intensity ratio for the $1022 \mathrm{~nm}$ and $1024 \mathrm{~nm}$ peaks slowly flatten out as these peaks almost gets hidden at elevated temperature (Fig. 3b). Hence, for these methods, accurate temperature estimation is only feasible in the $78-200 \mathrm{~K}$ interval, as we will see in upcoming section as well. Note that, as an example, our measured temperature variation of $1022 / 1027 \mathrm{~nm}$ intensity ratio is similar but different than earlier reports $[10,19]$. This again shows the need to take these calibration data carefully in a setting similar to the intended usage of the Yb:YAG sample (so universal calibration data are not feasible).

Figure $5 \mathrm{~b}$ shows the measured variation of the FWHM of the main $1030 \mathrm{~nm}$ emission peak with temperature. With increasing temperature, as expected, the FWHM of the line increases. As the spectra gets boarder, at around $250 \mathrm{~K}$, the main $1030 \mathrm{~nm}$ line combines with the side emission peaks and there is a sudden increase of measured FWHM value. The measured variation of FWHM is in very good 

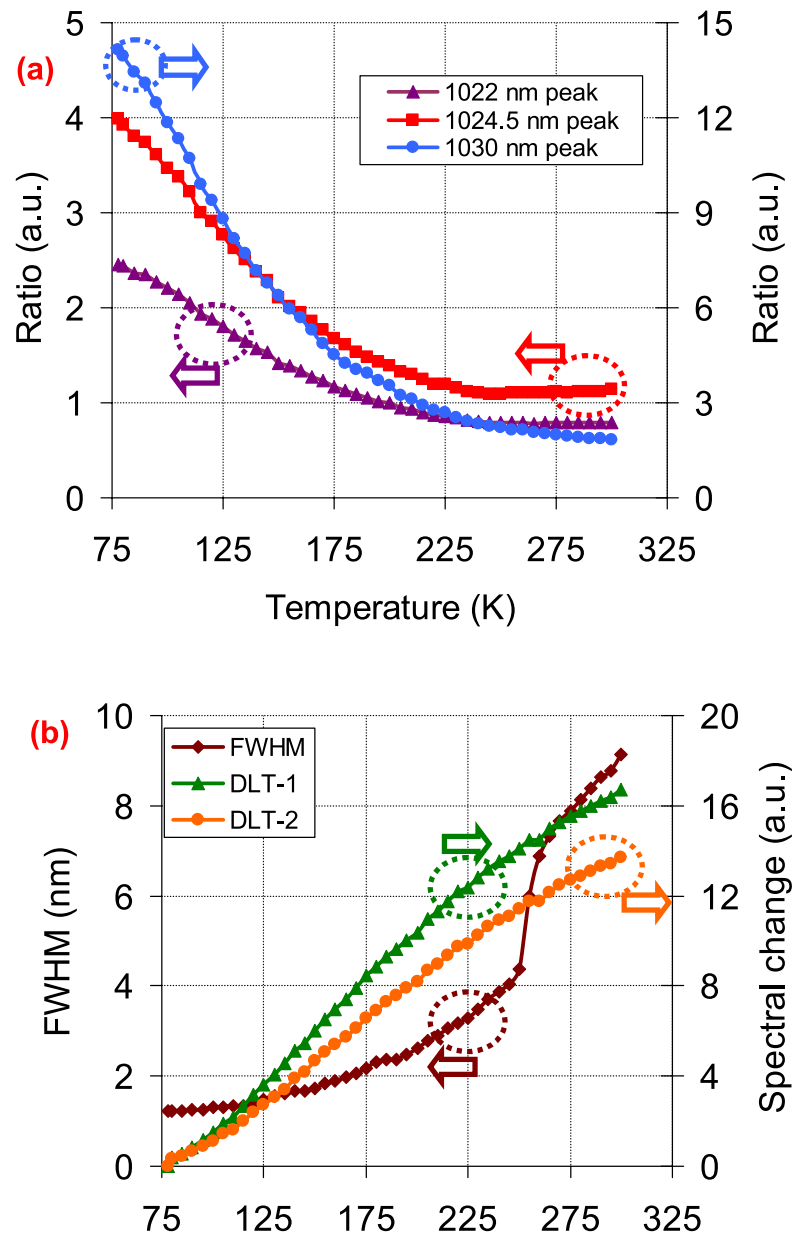

Temperature $(\mathrm{K})$

Fig. 5 a Measured variation of the ratio of intensity of the main $\sim 1030 \mathrm{~nm}$ peak to the intensity of $\sim 1027 \mathrm{~nm}$ dip (ratio of point 1-2 in Fig. 3), the intensity of the side $\sim 1024.5 \mathrm{~nm}$ peak to the intensity of $\sim 1027 \mathrm{~nm}$ dip (ratio of point 3-2 in Fig. 3), and the intensity of the side $\sim 1022 \mathrm{~nm}$ peak to the intensity of $\sim 1027 \mathrm{~nm}$ dip (ratio of point 4-2 in Fig. 3) with temperature. b Measured variation of the FWHM of the main $\sim 1030 \mathrm{~nm}$ peak with temperature. The calculated variation of integrated absolute change in spectral shape is also shown (DLT method), where the $78 \mathrm{~K}$ spectrum is used as a baseline. DLT-1 and DLT-2 investigate spectral changes in the 950-1060 nm and $950-1025 \mathrm{~nm}$ intervals; respectively

agreement with the previous reports by $[34,35]$, but do not exactly match. This shows that, from one perspective, the FWHM of the emission is not a strong function of system conditions (doping level of crystal, measurement conditions, etc....), and hence, unlike the intensity peak ratios, once a calibration curve is taken, it can potentially be used in different configurations. On the other hand, lasing and gain narrowing occurring in this line will certainly become an issue limiting wider usability of this method [10]. As usual, to achieve the minimum error bar in temperature estimation, the reference data should be repeated for different cases.
Figure $5 \mathrm{~b}$ also shows the measured variation of integrated overall absolute spectral change with temperature

$S_{\text {DLT }}\left(T, T_{0}\right)=\int\left|\Delta S\left(\lambda, T, T_{0}\right)\right| \mathrm{d} \lambda$.

As mentioned earlier, we have analyzed two different spectral regions in DLT: $950-1060 \mathrm{~nm}$ for DLT-1 and 950-1025 nm for DLT-2. Note that among the six methods investigated for temperature estimation, DLT method is the most sensitive one: if it is taken carefully, it can provide the most accurate temperature estimation. However, if the reference data are taken at a different condition than in the temperature measurement, it will be the most error-prone method. As mentioned earlier, with other methods, one is less sensitive in conditions: a slightly shifted beam on the crystal will not significantly change the measured FWHM of a line, but it can significantly modify the DLT signal creating large error bars.

\section{Error analysis for the different temperature estimation methods}

As we have stated earlier, we have used around 100 of the 630 emission spectra we took for obtaining the temperature calibration curves that is presented in the earlier section. To investigate the temperature estimation accuracy of different methods, we have randomly chosen spectra at known temperatures from the remaining data set, and checked how well the models work.

Figure 6a shows the estimated and measured temperatures using all the six methods. In Fig. 6b, we also show the errors in temperature estimation, defined as the difference between the measured and estimated temperature. A summary of error estimation performance of different methods is also given in Table 1, where we have listed the standard deviation of temperature estimation errors. In our analysis, we have looked at two ranges (78-200 K and 78-300 K), since as we mentioned earlier, some of the methods are not suitable for temperature estimation above $200 \mathrm{~K}$ due to disappearance of peaks at high temperatures (as it is clear from Fig. 6a).

When we compare all the methods, we see that both of the DLT methods enable temperature prediction with $\pm 1 \mathrm{~K}$ accuracy in the whole temperature range. DLT-1, which analyses a broader spectral range for temperature estimation, has a slightly better performance compared to DLT2. On the other hand, as discussed earlier, DLT-2 does not include spectra above $1025 \mathrm{~nm}$, where gain narrowing effects could be present, and it might be more suitable for high-power pumped systems. Among all the methods, the FWHM method has the largest error bar $( \pm 4 \mathrm{~K})$, and for our case, the error was due to the limited resolution of 

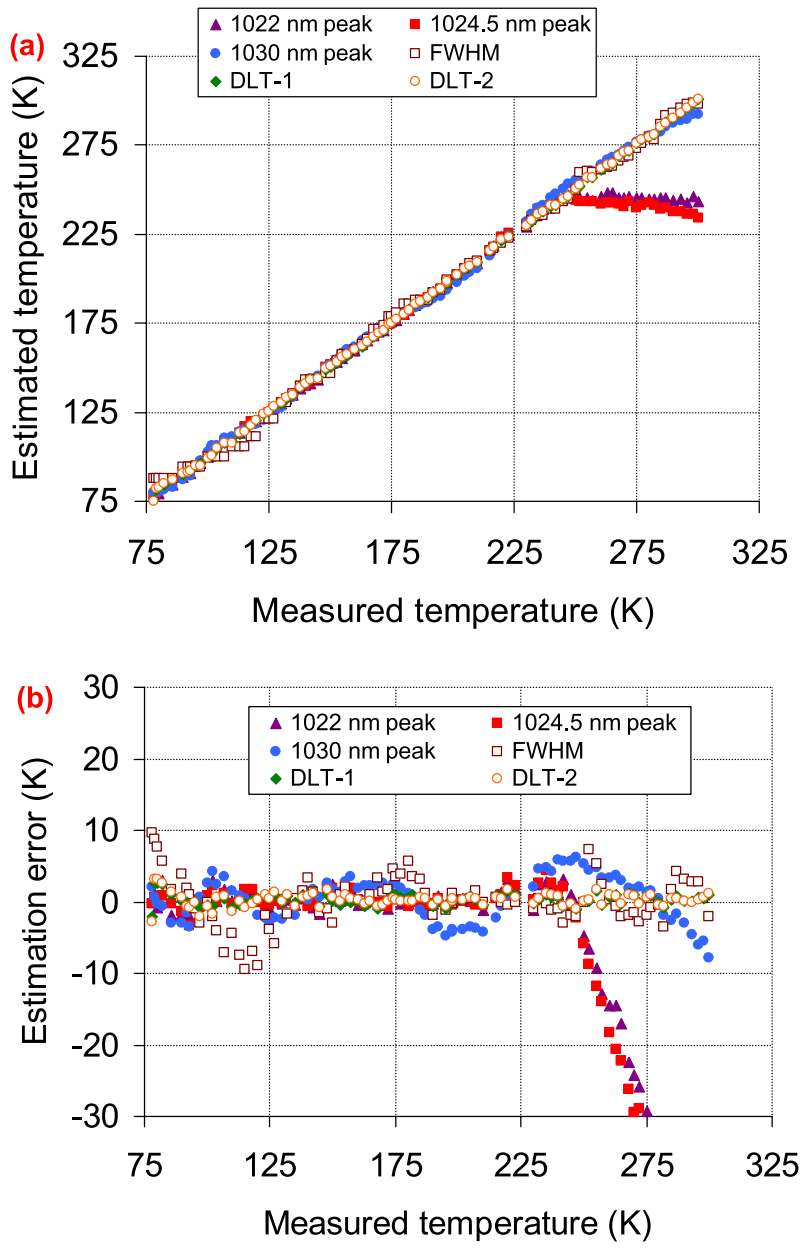

Fig. 6 a Measured and estimated temperature of Yb:YAG crystal using different methods. b Calculated estimation error in Yb:YAG crystal temperature for the different methods employed

Table 1 Comparison of temperature estimation accuracy of the different techniques employed for Yb:YAG. Standard deviation (SD) of errors is given in the table for comparison

\begin{tabular}{lll}
\hline Estimation method & $\begin{array}{l}\text { Error } \\
(78-200 \mathrm{~K})\end{array}$ & $\begin{array}{l}\text { Error } \\
(78-300 \mathrm{~K})\end{array}$ \\
\hline $1022 \mathrm{~nm}$ ratio & 1.1 & - \\
$1024.5 \mathrm{~nm}$ ratio & 1.0 & - \\
$1030 \mathrm{~nm}$ ratio & 2.4 & 3.1 \\
FWHM & 4.0 & 3.4 \\
DLT-1 & 0.9 & 0.8 \\
DLT-2 & 1.1 & 0.9 \\
\hline
\end{tabular}

the spectrometer $(\sim 0.1 \mathrm{~nm})$ : the FWHM information was digitized in discrete steps of $\sim 0.05 \mathrm{~nm}$ and this already creates an additional error bar of $\pm 2-3 \mathrm{~K}$. Hence, we believe that the FWHM method accuracy could be significantly increased by employing a spectrometer with a higher resolution.
Among the methods that are based on looking at the relative intensity of the emission peaks, despite its larger slope (larger change of intensity with temperature), interestingly, the temperature estimation based on the $1030 / 1027 \mathrm{~nm}$ peak ratio had a significantly higher error bar $( \pm 3 \mathrm{~K})$ compared to the others. We believe that this might be due to the sensitivity of this peak to gain modification as discussed in earlier literature as well [10]. The other methods $(1022 / 1027 \mathrm{~nm}$ ratio and 1024.5/1027 nm ratio) work better with a temperature estimation error of $\pm 1 \mathrm{~K}$ in the $78-200 \mathrm{~K}$ interval.

\section{Sample temperature measurements}

In the earlier section, we have analyzed temperature estimation accuracy for the different methods. Here, we will present two sample temperature measurements that has practical importance for us: (1) for a Yb:YAG rod soldered onto a copper heat sink that is cooled by boiling liquid nitrogen, and (2) the same rod in cw lasing experiments. In the first case, the temperature measurement of the rod enables a quality checking mechanism for the thermal contact quality of the rod. For the second case, a real-time measurement of crystal temperature during lasing enables optimization of laser performance.

As an example, Fig. 7a shows the estimated average temperature of a $24 \mathrm{~mm}$-long $1 \% \mathrm{Yb}$-doped YAG sample, as a function of absorbed pump power level in a non-lasing geometry. All six methods have been used for temperature estimation, and at least three data points are taken for each absorbed pump power level, and the error bars in the graph show the standard deviation of the measurements. As we see, the error bars are hardly visible for most data points, showing the repeatability of the temperature estimation. Note that all the methods estimate a very similar temperature trend. To compare, in Fig. 7b, we also show the average estimated temperature of the Yb:YAG crystal using all the methods, and the error bar now shows the standard deviation of temperature estimation using different methods. The average standard deviation for temperature estimation is only $2.25 \mathrm{~K}$, which shows the good match between different temperature estimation methods employed in this work.

In Fig. 7b, for comparison purposes, we have also included numerically calculated variation of the average temperature of the Yb:YAG crystal with absorbed pump power [36]. The numerical estimation is performed for three different assumptions for fractional thermal load (FTL): (a) ideal case where the FTL is same as the quantum defect (QD: 8.5\%: 1-940/1030), (b) FTL is 1.5 times the QD, and (c) FTL is two times the QD. Note that, in general, the measured trend of temperature increase match relatively well to the calculated trends. We also see that the measured temperatures are below what is estimated for $2 \times \mathrm{QD}$ case: 

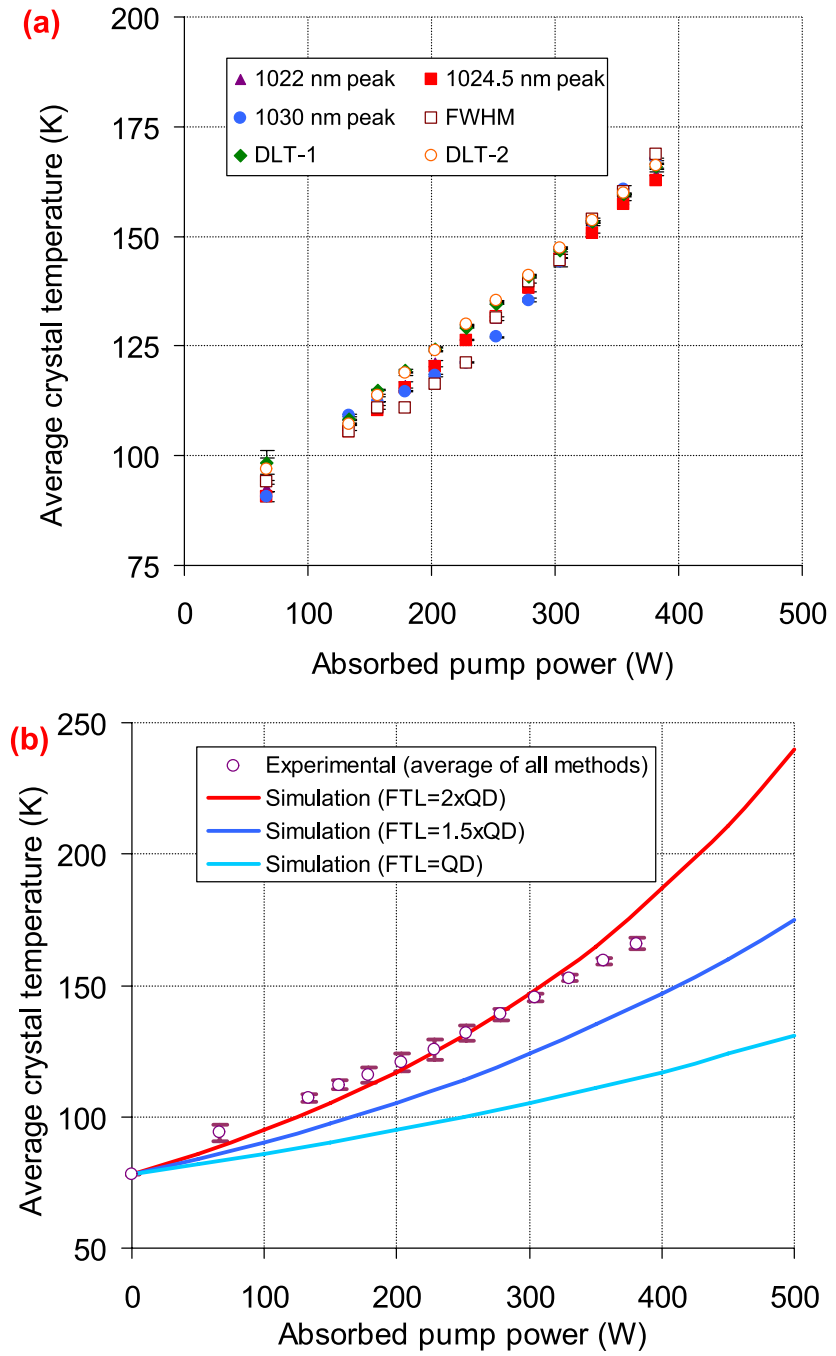

Fig. 7 a Estimated average temperature of $1 \%$ Yb-doped YAG crystal as a function of absorbed pump power using the six different temperature estimation methods that are outlined earlier. At each pump power level, a minimum of three spectra is collected, and the error bars in the data indicate the standard deviation of estimation for each method. b Temperature estimation curve obtained by averaging all the six methods (shown with open markers). The solid curve shows the calculated increase in crystal temperature using COMSOL, based on a model similar to what is described in [36]. The calculation has been performed for a fractional thermal load (FTL) of 1, 1.5, and 2 times the quantum defect (QD)

indicating that the fractional thermal load should be lower than $2 \times \mathrm{QD}$. This is in agreement with the direct thermal load measurement of Fan, who reported a fractional thermal load of around $11 \%(1.3 \times \mathrm{QD})$ in a room-temperature Yb:YAG system [37]. In our experiments with Yb:YLF, we have seen that, in the samples with the best thermal contact quality (bonding quality), the measured temperatures could get very close to simulation results assuming $1.5 \times \mathrm{QD}[23$, 36]. Here, with this Yb:YAG sample we are a little above

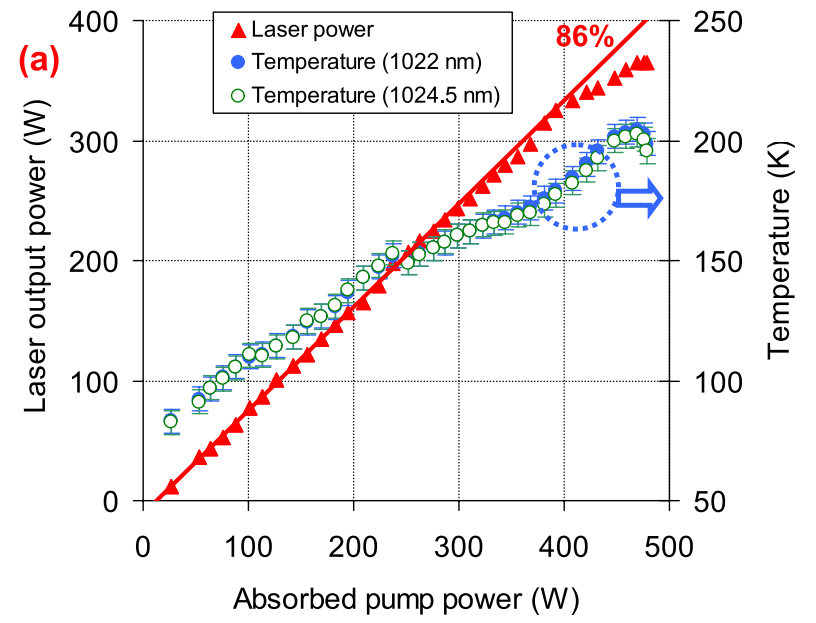

(b)
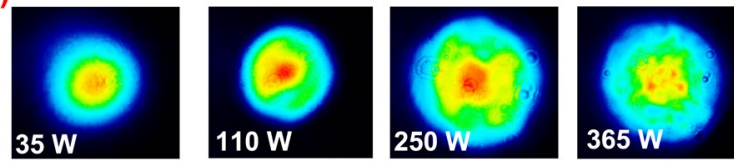

Fig. 8 a Measured variation of cryogenic Yb:YAG cw laser performance. Estimated variation of average Yb:YAG crystal temperature with absorbed pump power level is also shown. Temperature estimation is performed using the $1022 \mathrm{~nm}$ and $1024.5 \mathrm{~nm}$ peak methods. The cw lasing data are taken using a 1\%-doped Yb:YAG crystal in a short flat-flat cw cavity using a 25\% output coupler. b Sample nearfield beam profiles of the short-cavity cw Yb:YAG cryogenic laser

that, and this we believe is due to the non-optimum bonding of this specific crystal.

As a second example, we have used the developed temperature estimation methods for real-time temperature probing during cryogenic lasing experiments with Yb:YAG. During lasing around $1030 \mathrm{~nm}$, the laser background signal modifies the emission spectra, and hence, most of the developed methods could not be used for accurate temperature estimation. On the other hand, the $1022 / 1027 \mathrm{~nm}$ ratio and $1024.5 / 1027 \mathrm{~nm}$ ratio method still provide trustable information as they are further away from the lasing peak. Figure 8 a summarizes performance of a $940 \mathrm{~nm}$ pumped continuous-wave Yb:YAG laser operating at $1030 \mathrm{~nm}$. The simple short-cavity described earlier in Fig. 2 is used for lasing. The data are taken with a $25 \%$ transmitting output coupler (OC). The $\mathrm{cw}$ laser had a lasing threshold of around $15 \mathrm{~W}$ and the laser provided up to $365 \mathrm{~W}$ of output power at an absorbed pump power level of around $480 \mathrm{~W}(520 \mathrm{~W}$ incident power and around $92 \%$ absorption). The corresponding slope efficiency with respect to absorbed pump power is as high as $86 \%$ (red solid line) and is very close to the quantum defect limit (91.2\%), indicating good mode-matching between the laser and pump beams. The sample output beam profile data 
are shown in Fig. 8b. The laser performance is limited by thermal lensing, and it is hence quite important to estimate the laser crystal temperature.

The temperature of the YAG crystal is estimated using both the $1022 / 1027 \mathrm{~nm}$ ratio and $1024.5 / 1027 \mathrm{~nm}$ ratio methods. The results obtained with each method are shown separately in Fig. 8a. We see that the temperature estimates with both methods are almost identical, and the rms value of the difference is only $1.2 \mathrm{~K}$. This indicates the good measurement accuracy of the system, and we estimate an overall maximum error margin of $\pm 5 \mathrm{~K}$ for this measurement.

Note that, the temperature increase with pump power is faster than the non-lasing case discussed above. Unfortunately, for this short flat-flat cavity, most of the transmitted pump power $(\sim 40 \mathrm{~W})$ is reflected back to the system by the output coupler creating additional heating of the crystal (the back reflected pump beam hitting back the crystal and the copper crystal holder is quite large in size and creates significant additional thermal load). This additional heat load is the reason for the faster temperature increase in this cavity compared to non-lasing case. Moreover, we want to note that the kink in the temperature estimation curve at around $250 \mathrm{~W}$ absorbed pump power is due to the realignment of the laser at that point, which shows that small temperature difference due to better extraction could also be easily measured with the proposed temperature estimation methods.

\section{Conclusion}

In this study, we have investigated six different temperature estimation methods for cryogenic Yb:YAG laser and amplifier systems. We have shown that for samples with a homogeneous temperature distribution in situ contactless optical temperature estimation within $\pm 1 \mathrm{~K}$ is feasible. We have further demonstrated that, for Yb:YAG rods pumped with $100 \mathrm{~s}$ of Watts of pump power, average crystal temperature could be estimated with better than $\pm 5 \mathrm{~K}$ accuracy for both lasing and non-lasing conditions.

Acknowledgements The authors acknowledge support from previous group members L. E. Zapata and K. Zapata for establishing the indiumbonding technology for YLF at CFEL-DESY. UD acknowledge support from BAGEP Award of the Bilim Akademisi.

Funding Open Access funding enabled and organized by Projekt DEAL. Seventh Framework Programme (FP7) FP7/2007- 2013 European Research Council (ERC) (609920).

\section{Declarations}

Conflict of interest The authors declare no conflicts of interest.

Open Access This article is licensed under a Creative Commons Attribution 4.0 International License, which permits use, sharing, adaptation, distribution and reproduction in any medium or format, as long as you give appropriate credit to the original author(s) and the source, provide a link to the Creative Commons licence, and indicate if changes were made. The images or other third party material in this article are included in the article's Creative Commons licence, unless indicated otherwise in a credit line to the material. If material is not included in the article's Creative Commons licence and your intended use is not permitted by statutory regulation or exceeds the permitted use, you will need to obtain permission directly from the copyright holder. To view a copy of this licence, visit http://creativecommons.org/licenses/by/4.0/.

\section{References}

1. P. Russbueldt, T. Mans, J. Weitenberg, H.D. Hoffmann, R. Poprawe, Compact diode-pumped $1.1 \mathrm{~kW}$ Yb:YAG Innoslab femtosecond amplifier. Opt. Lett. 35(24), 4169-4171 (2010)

2. D.J. Ripin, J.R. Ochoa, R.L. Aggarwal, T.Y. Fan, 300-W cryogenically cooled Yb:YAG laser. IEEE J. Quantum Electron. 41(10), 1274-1277 (2005)

3. M. Ganija, D. Ottaway, P. Veitch, J. Munch, Cryogenic, high power, near diffraction limited, Yb:YAG slab laser. Opt. Express 21(6), 6973 (2013)

4. A. Giesen, J. Speiser, Fifteen years of work on thin-disk lasers: results and scaling laws. IEEE J. Sel. Top. Quantum Electron. 13(3), 598-609 (2007)

5. C. Baumgarten, M. Pedicone, H. Bravo, H. Wang, L. Yin, C.S. Menoni, J.J. Rocca, B.A. Reagan, $1 \mathrm{~J}, 05 \mathrm{kHz}$ repetition rate picosecond laser. Opt. Lett. 41(14), 3339 (2016)

6. J.-P. Negel, A. Loescher, A. Voss, D. Bauer, D. Sutter, A. Killi, M.A. Ahmed, T. Graf, Ultrafast thin-disk multipass laser amplifier delivering $14 \mathrm{~kW}(47 \mathrm{~mJ}, 1030 \mathrm{~nm})$ average power converted to $820 \mathrm{~W}$ at $515 \mathrm{~nm}$ and $234 \mathrm{~W}$ at $343 \mathrm{~nm}$. Opt. Express 23(16), 21064 (2015)

7. T. Dietz, M. Jenne, D. Bauer, M. Scharun, D. Sutter, A. Killi, Ultrafast thin-disk multi-pass amplifier system providing 1.9 $\mathrm{kW}$ of average output power and pulse energies in the $10 \mathrm{~mJ}$ range at $1 \mathrm{ps}$ of pulse duration for glass-cleaving applications. Opt. Express 28(8), 11415-11423 (2020)

8. U. Demirbas, J. Thesinga, H. Cankaya, M. Kellert, F.X. Kartner, M. Pergament, High-power passively mode-locked cryogenic Yb:YLF laser. Opt. Lett. 45(7), 2050-2053 (2020)

9. D.J. Ripin, J.R. Ochoa, R.L. Aggarwal, T.Y. Fan, 165-W cryogenically cooled Yb:YAG laser. Opt. Lett. 29(18), 2154 (2004)

10. H. Chi, K.A. Dehne, C.M. Baumgarten, H.C. Wang, L. Yin, B.A. Reagan, J.J. Rocca, In situ 3-D temperature mapping of high average power cryogenic laser amplifiers. Opt. Express 26(5), 5240-5252 (2018)

11. H. Chi, C.M. Baumgarten, E. Jankowska, K.A. Dehne, G. Murray, A.R. Meadows, M. Berrill, B.A. Reagan, J.J. Rocca, Thermal behavior characterization of a kilowatt-power-level cryogenically cooled Yb:YAG active mirror laser amplifier. J. Opt. Soc. Am. B 36(4), 1084 (2019)

12. J. Koerner, C. Vorholt, H. Liebetrau, M. Kahle, D. Kloepfel, R. Seifert, J. Hein, M.C. Kaluza, Measurement of temperature-dependent absorption and emission spectra of $\mathrm{Yb}: \mathrm{YAG}$, $\mathrm{Yb}: \mathrm{LuAG}$, and $\mathrm{Yb}: \mathrm{CaF} \_2$ between $20{ }^{\circ} \mathrm{C}$ and $200{ }^{\circ} \mathrm{C}$ and 
predictions on their influence on laser performance. J. Opt. Soc. Am. B 29(9), 2493 (2012)

13. J.L. Battaglia, B. Gavory, A. Courjaud, Thermal contact resistance measurement at the interface between $\mathrm{Yb}$ :YAG crystal and WCu cooler at $80 \mathrm{~K}$ and $300 \mathrm{~K}$. J. Appl. Phys. 120(19), 195102 (2016)

14. S. Chénais, F. Druon, S. Forget, F. Balembois, P. Georges, On thermal effects in solid-state lasers: the case of ytterbium-doped materials. Prog. Quantum Electron. 30(4), 89-153 (2006)

15. S. Chenais, S. Forget, F. Druon, F. Balembois, P. Georges, Direct and absolute temperature mapping and heat transfer measurements in diode-end-pumped Yb : YAG. Appl. Phys. B Lasers Opt. 79(2), 221-224 (2004)

16. J. Petit, B. Viana, P. Goldner, Internal temperature measurement of an ytterbium doped material under laser operation. Opt. Express 19(2), 1138 (2011)

17. C. Xu, Y. Huang, Y. Lin, J. Huang, X. Gong, Z. Luo, Y. Chen, Real-time measurement of temperature distribution inside a gain medium of a diode-pumped $\mathrm{Er}^{\wedge} 3+/ \mathrm{Yb}^{\wedge} 3+155 \mu \mathrm{m}$ laser. Opt. Lett. 42(17), 3383 (2017)

18. H.-J. Moon, C. Lim, G.-H. Kim, U. Kang, Study of operation dynamics for crystal temperature measurement in a diode endpumped monolithic Yb:YAG laser. Opt. Express 21(25), 31506 (2013)

19. H. Furuse, J. Kawanaka, N. Miyanaga, H. Chosrowjan, M. Fujita, K. Takeshita, Y. Izawa, Output characteristics of high power cryogenic Yb:YAG TRAM laser oscillator. Opt. Express 20(19), 21739-21748 (2012)

20. S. Tokita, J. Kawanaka, M. Fujita, T. Kawashima, Y. Izawa, Sapphire-conductive end-cooling of high power cryogenic Yb:YAG lasers. Appl. Phys. B Lasers Opt. 80(6), 635-638 (2005)

21. D.A. Cirovic, Feed-forward artificial neural networks: applications to spectroscopy. Trends Anal. Chem. 16(3), 148-155 (1997)

22. V.V. Petrov, G.V. Kuptsov, A.I. Nozdrina, V.A. Petrov, A.V. Laptev, A.V. Kirpichnikov, E.V. Pestryakov, Contactless method for studying temperature within the active element of a multidisk cryogenic amplifier. Quantum Electron. 49(4), 358-361 (2019)

23. U. Demirbas, J. Thesinga, M. Kellert, F.X. Kärtner, M. Pergament, Comparison of different in situ optical temperature probing techniques for cryogenic Yb:YLF. Opt. Mater. Express 10(12), 3403-3413 (2020)

24. U. Demirbas, M. Kellert, J. Thesinga, Y. Hua, S. Reuter, F.X. Kärtner, M. Pergament, Comparative investigation of lasing and amplification performance in cryogenic Yb:YLF systems. Appl. Phys. B 127(3), 46 (2021)

25. M. Kellert, U. Demirbas, J. Thesinga, S. Reuter, M. Pergament, F.X. Kärtner, High power (> 500W) cryogenically cooled $\mathrm{Yb}$ :YLF cw-oscillator operating at $995 \mathrm{~nm}$ and $1019 \mathrm{~nm}$ using E//c axis for lasing. Opt. Express 29(8), 11674 (2021)
26. S.D. Melgaard, A.R. Albrecht, M.P. Hehlen, M. Sheik-Bahae, Solid-state optical refrigeration to sub-100 Kelvin regime. Sci. Rep. 6, 20380 (2016)

27. D.V. Seletskiy, S.D. Melgaard, S. Bigotta, A. Di Lieto, M. Tonelli, M. Sheik-Bahae, Laser cooling of solids to cryogenic temperatures. Nat. Photon. 4(3), 161-164 (2010)

28. A. Pant, X.J. Xia, E.J. Davis, P.J. Pauzauskie, Solid-state laser refrigeration of a composite semiconductor $\mathrm{Yb}: \mathrm{YLiF} 4$ optomechanical resonator. Nat. Commun. 11(1), 3235 (2020)

29. A. Volpi, A. Di Lieto, M. Tonelli, Novel approach for solid state cryocoolers. Opt. Express 23(7), 8216-8226 (2015)

30. E.S.L. de Filho, G. Nemova, S. Loranger, R. Kashyap, Laserinduced cooling of a $\mathrm{Yb}$ :YAG crystal in air at atmospheric pressure. Opt. Express 21(21), 24711 (2013)

31. M. Pergament, L.E. Zapata, U. Demirbas, Y. Liu, M. Kellert, S. Reuter, J. Thesinga, Y. Hua, H. Cankaya, A.-L. Calendron, F.X. Kärtner, High energy cryogenic Yb:YAG and Yb:YLF chirped pulse amplifiers. In: Laser Congress 2020 (ASSL, LAC) (2020), Paper ATu4A.3 (The Optical Society, 2021), p. ATu4A.3

32. L.E. Zapata, H. Lin, A.L. Calendron, H. Cankaya, M. Hemmer, F. Reichert, W.R. Huang, E. Granados, K.H. Hong, F.X. Kartner, Cryogenic Yb:YAG composite-thin-disk for high energy and average power amplifiers. Opt. Lett. 40(11), 2610-2613 (2015)

33. L.E. Zapata, F. Reichert, M. Hemmer, F.X. Kartner, $250 \mathrm{~W}$ average power, $100 \mathrm{kHz}$ repetition rate cryogenic $\mathrm{Yb}$ :YAG amplifier for OPCPA pumping. Opt. Lett. 41(3), 492-495 (2016)

34. I.B. Mukhin, O.V. Palashov, E.A. Khazanov, A.G. Vyatkin, E.A. Perevezentsev, Laser and thermal characteristics of $\mathrm{Yb}$ : YAG crystals in the $80-300 \mathrm{~K}$ temperature range. Quantum Electron. 41(11), 1045-1050 (2011)

35. J. Körner, V. Jambunathan, J. Hein, R. Seifert, M. Loeser, M. Siebold, U. Schramm, P. Sikocinski, A. Lucianetti, T. Mocek, M.C. Kaluza, Spectroscopic characterization of Yb3+-doped laser materials at cryogenic temperatures. Appl. Phys. B Lasers Opt. 116(1), 75-81 (2014)

36. U. Demirbas, H. Cankaya, J. Thesinga, F.X. Kärtner, M. Pergament, Power and energy scaling of rod-type cryogenic $\mathrm{Yb}: \mathrm{YLF}$ regenerative amplifiers. J. Opt. Soc. Am. B 37(6), 1865-1877 (2020)

37. T.Y. Fan, Heat generation in Nd:YAG and Yb:YAG. IEEE J. Quantum Electron. 29, 1457-1459 (1993)

Publisher's Note Springer Nature remains neutral with regard to jurisdictional claims in published maps and institutional affiliations. 